THERAPEUTICS

\section{Taking a bite out of tumours}

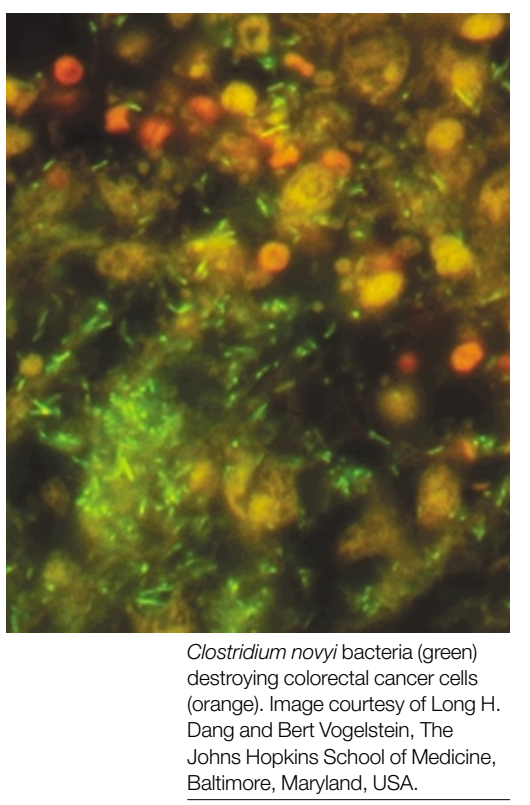

Although many drugs are effective in killing rapidly dividing cancer cells, it is difficult to deliver these drugs to the poorly vascularized, hypoxic regions inside large tumours. Bert Vogelstein's group at Johns Hopkins University have developed an 'insideout' therapy to destroy the oxygendeprived necrotic areas of tumours — using meat-eating bacteria.

Although tumours induce formation of new blood vessels to deliver nutrients and oxygen to the growing tumour, angiogenesis does not keep pace with the growth of the neoplastic cells. This results in large hypoxic areas throughout the tumour. Cancer cells in these areas are not killed by ionizing radiation, which depends on oxygen, or by chemotherapeutic drugs, which do not reach these regions.
Reporting in Proceedings of the National Academy of Sciences, Long Dang et al. report an attempt to take advantage of the fact that necrotic tissues exist only in tumours and not in normal tissues. They performed a screen for anaerobic bacteria that can distribute throughout poorly vascularized regions of tumours. One of the strains they isolated, Clostridium novyi (which the authors grew on cooked meat particles) fulfilled this criterion, but was also, unfortunately, toxic to the mice. As this bacterium carries its toxin gene on a phage episome, the authors were able to isolate phagefree strains that no longer killed mice. But would these bacteria still be able to kill tumour cells?

The authors tested the ability of intravenously administered C. novyi spores to destroy colorectal tumours in a mouse xenograft model. The spores were administered with conventional chemotherapeutic agents, in the hope of killing the vascularized cancer cells located on the tumour's outside
- as well as the hypoxic regions inside. Treatment with chemotherapy alone usually only slows, but does not stop, tumour growth. The combination bacteriolytic therapy (COBALT), however, caused the tumours to become black necrotic masses that shrank and disappeared, whereas surrounding normal tissue remained intact. More than three-quarters of the tumours treated, including very large tumours, were completely destroyed within 24 hours, and approximately half the mice were cured with no evidence of tumour regrowth. Similar results were seen with melanomas grown in C57BL/6 mice.

Interestingly, the authors found that co-administration of the bacterial spores with D10, a chemotherapeutic drug that acts by collapsing the tumour vasculature, was the most effective antitumour combination. The authors believe that the D10-induced vascular collapse further lowers the oxygen tension near the bacteria and increases their proliferation and activity.

\title{
Fishing for trouble
}

\begin{abstract}
Helicobacter pylori is a bacterium that is present in the gastrointestinal tract of about half the world's human population and is believed to cause gastric cancer. $H$. pylori strains carrying the cagA gene are known to be particularly virulent, but little is known about CagA function. Reporting in Science, Hideaki Higashi and colleagues now describe a protein-fishing expedition that led to the discovery of what might be a transformationinducing CagA target.

H. pylori strains that carry the cagA gene are nasty creatures that attach to host gastric epithelial cells and inject CagA protein. Inside the cell, $\mathrm{CagA}$ is

phosphorylated and triggers morphological changes similar to those induced by growth factors. But how can one bacterial protein activate cell shape change?

To find out how CagA operates, Higashi et al. went fishing for proteins that interact with it. They transfected human gastric epithelial cells with haemagglutinin-tagged CagA, and also with a mutant form that could not be phosphorylated. As predicted, expression of
\end{abstract}

wild-type, but not phosphorylation-resistant, CagA induced cellular elongation and spreading. Immunoprecipitation experiments revealed that the wild type but not the phosphorylation-resistant protein bound a protein called SHP-2. SHP-2 is a protein tyrosine phosphatase that is known to positively regulate signal transduction events from a variety of activated receptor tyrosine kinases. SHP-2 has also been shown to activate cell migration and adhesion.

SHP-2's phosphatase activity seems to be required for cell morphological changes. Treatment of CagA-expressing gastric epithelial cells with phosphatase inhibitors prevented induction of cellular spreading and elongation. Higashi et al. also created a phosphatase-defective form of SHP-2 that was still able to interact with CagA, and found that co-transfection of cells with this mutant, along with CagA, did not induce cell spreading.

So, if the phosphatase activity of SHP-2 is required to induce cell shape changes, what does CagA do? Because CagA is membrane associated, the authors proposed that the role of CagA might be to recruit SHP-2 to the plasma membrane. Accordingly, expression of a membrane-targeted, constitutively active form of SHP-2 was able to induce cell morphological changes in the absence of CagA. This indicates that SHP-2 acts at the plasma membrane to induce cell shape change, and its activation might be an important component of gastric tumorigenesis. It also provides an explanation for numerous epidemiological studies showing that $\operatorname{cag} A^{+}$strains are more carcinogenic than $\operatorname{cag} A^{-}$strains.

Kristine Novak

(2) References and links

ORIGINAL RESEARCH PAPER Higashi, H. et al. SHP-2 tyrosine phosphatase as an intracellular target of Helicobacter pylori CagA protein. Science 2001 Dec 13; [epub ahead of print] FURTHER READING Peek, R. M. Jr and Blaser, M. J. Helicobacter pylori and gastrointestinal tract adenocarcinomas. Nature Rev. Cancer 2, 28-37 (2002)

WEB SITE

Hideaki Higashi's lab:

http://www.imm.hokudai.ac.jp/others/staff/staff-e.html 